\title{
CONDICIONES DE TRABANO EN LA MICRO PEQUEÑA Y MEDIANA EMPRESA. RETO DEL TRABAJO DECENTE EN UNA ECONOMÍA EN PANDEMIA'
}

\author{
Working conditions in Micro Small and Medium Enterprises. Decent Work Challenge in a \\ Pandemic Economy
}

Leidy Stefany Torres Pinto

Itorres@poligran.edu.co

Lisbeth Marina Molero Suarez

Imolero@poligran.edu.co

Allyson Karen Venegas Camargo

vcallynson@poligran.edu.co

Institución Universitaria Politécnico Grancolombiano

Colombia

\begin{abstract}
Resumen
Este artículo presenta el análisis de la primera dimensión condiciones de trabajo en las micro, pequeñas y medianas empresas del sector servicios en la ciudad de Bogotá, debido a su carácter distintivo para lograr la sostenibilidad en el entorno laboral y en respuesta a los desafíos presentes el contexto económico, sanitario y social, planteado a partir de la aparición del virus SARS-COV-2. En lo que corresponde al aspecto metodológico la investigación responde a un estudio de tipo cuantitativo, analítico y descriptivo de las condiciones de trabajo en el contexto actual, cuya muestra estuvo conformada por 30 (treinta) MiPymes del sector servicios a las cuales se les aplicó un instrumento donde los 18 primeros ítems corresponden a las dimensiones que configuran actualmente dichas condiciones en la población abordada se intenta generar un espacio para la reflexión y la generación de conciencia en relación con el bienestar laboral y la calidad de vida que en la actualidad tienen los individuos en sus sitios de trabajo abriendo un camino para la generación de estrategias en lo que respecta a las condiciones de trabajo presentes en la relación laboral, convirtiendo éstas en una ventaja competitiva para el sector. El estudio permitió reconocer las condiciones actuales puntualizando aquellas que van en detrimento de la relación laboral como el estrés y la salud en el entorno.
\end{abstract}

Palabras clave:

Condiciones de trabajo, trabajo decente, accidente laboral

\footnotetext{
${ }^{1}$ El presente artículo representa el primer avance de investigación del proyecto de investigación titulado: trabajo decente perspectiva estratégica reto en una economía en pandemia para las MiPymes. 


\begin{abstract}
This article presents the analysis of the first dimension working conditions in the micro, small and medium enterprises of the service sector in the city of Bogotá, due to its distinctive character to achieve sustainability in the work environment and in response to the challenges present in the economic, health and social context, raised from the appearance of the SARS-COV-2 virus. Regarding the methodological aspect, the research responds to a quantitative, analytical and descriptive study of the working conditions in the current context, whose sample was made up of 30 (thirty) MSMEs from the service sector to which a instrument where the first 18 items correspond to the dimensions that currently configure these conditions in the population addressed, an attempt is made to generate a space for reflection and the generation of awareness in relation to work well-being and the quality of life that individuals currently have in their workplaces, opening a way for the generation of strategies regarding the working conditions present in the employment relationship, turning these into a competitive advantage for the sector. The study allowed us to recognize current conditions, specifying those that are detrimental to the employment relationship, such as stress and health in the environment.
\end{abstract}

Recepción: $\quad 16.02 .2021$

Aceptación: $\quad 11.05 .2021$

Cite este artículo como: Torres-Pinto, L., Molero-Suárez, L., Venegas-Camargo, A. (2021). Condiciones de trabajo en la micro, pequeña y mediana empresa. Reto del trabajo decente en una economía en pandemia. Working papers FNGS, 2 (2), pp. 1-11. 


\section{INTRODUCCIÓN}

En la actualidad el mercado laboral se ha visto golpeado gravemente por la afección pandémica a nivel mundial, las pequeñas y medianas empresas del sector servicios no escapan a los efectos adversos de la pandemia. Como producto del desequilibrio a nivel económico y social se genera insatisfacción en el ámbito laboral que repercute en el trabajo decente. Según la Organización Internacional del Trabajo (OIT, 2014) el trabajo decente se vincula con los deseos y aspiraciones del individuo durante su trayectoria laboral, la cual equipara las diferentes oportunidades de trabajo productivo. En estos momentos estos deseos y aspiraciones se ven afectados, ya que las organizaciones y su personal deben asumir postura de adaptabilidad y resiliencia.

De igual manera las condiciones de trabajo representan un aspecto determinante para lograr la productividad sobre todo en las MiPymes, las cuales están expuestas y en gran medida afectadas producto de la pandemia por COVID-19. Además de las necesidades que tradicionalmente tienen las MiPymes se suma todo el escenario actual que hace necesario dar una mirada a las condiciones que actualmente ofrecen a los individuos específicamente las pymes de sector servicios. Ya que es uno de los sectores más afectados por la situación actual. Así mismo, mediante este avance de investigación se busca reflejar de manera parcial las dimensiones del trabajo decente que hoy más que nunca cobran un papel protagónico en lo que corresponde a las condiciones de trabajo ya que estas involucran las condiciones de seguridad, que a su vez, representan un indicador de suma importancia para la operatividad de las empresas que se mantiene en el mercado pese a la situación que se vive a nivel mundial.

Las condiciones de trabajo involucran elementos determinantes como son las condiciones psicológicas las cuales se han visto afectadas producto de la incertidumbre del nuevo escenario caracterizado por el confinamiento que ha llevado a un gran porcentaje de empresas al teletrabajo y a un desequilibrio de las jornadas de trabajo, lo que constituye una condición excepcional de prestación de los servicios.

Este avance muestra, de manera descriptiva, la situación de un grupo de empresas que hasta la fecha ha aceptado la revisión de las dimensiones contentivas del trabajo decente para lograr obtener estrategias efectivas que les permitan fortalecer su relación con sus colaboradores.

\section{Trabajo Decente}

Larani (2015) siguiendo a Juan Somavia mantiene la postura de que el trabajo decente está enfocado a la acción productiva remunerada y que basada en la realidad del COVID-19, debe adecuarse a las alternativas ocupacionales adaptadas a la realidad en el marco de la tecnología donde el ejercicio productivo se ubica en la capacidad digital que posea el personal, el teletrabajo pasa a ser una opción como otras y se ejerce con la individualidad de demostrar las condiciones de libertad, equidad y respeto a la dignidad humana, proyectando las capacidades para adaptarse a los cambios exigidos en el mercado actual.

Lo planteado anteriormente en relación con el trabajo decente sustenta la preocupación prioritaria sobre el elevado número de desempleados, y pone en un segundo plano aspectos como la necesidad de trabajo digno con ingresos atractivos. Así, para la Dirección General de la OIT (2012, Cita Segovia 2016); esta pandemia distorsiona sus planteamientos, pues afecta la productividad laboral, la protección de los derechos del trabajador, la generación de ingresos suficientes para una seguridad social adecuada, con oportunidades en el trabajo y protección familiar, con proyecciones personales y profesionales y la igualdad que, aunque reconocida, no es sustancialmente considerada prioridad, por la tendencias gubernamental de enfocar sus intereses en el sector salud, asistencial, educativo y de información. 
Por ello, Larani (2015) siguiendo a Juan Somavia afirma que con base en la realidad del COVID-19, es necesario adecuarse a las alternativas ocupacionales adaptadas a la realidad en el marco de la tecnología donde el ejercicio productivo se ubica en la capacidad digital que posea el personal, el teletrabajo pasa a ser una opción como otras y se ejerce con la individualidad de demostrar las condiciones de libertad, equidad y respeto a la dignidad humana, proyectando las capacidades para adaptarse a los cambios exigidos en el mercado actual.

\section{Condiciones de Trabajo}

Ante la situación sanitaria, las condiciones laborales son diferentes en la medida en que las organizaciones presentan posturas adaptativas que muchas veces no son asumidas por el personal que labora en las empresas por no adaptarse a los cambios y mucho menos de forma brusca. De esta manera, para la Organización Internacional del Trabajo (OIT, 2014) la visión integradora empresa-recurso-humana es vital para el equilibrio productivo en el que el medio social, físico y cultural, busca brindar una calidad de vida adecuada. No obstante, en la perturbación de salud existente no se propicia una vida laboral que coincida con lo establecido.

Es por ello, que el cambio abrupto en el escenario laboral ha generado opciones diversas por parte del empleador que le permita mantener al personal activo considerando el trabajo remoto (en casa) como una de las formas más adecuada, considerando siempre la postura y la opinión del trabajador; de acuerdo con lo planteado por Monasterio, Villamizar y Asociados (2015) los beneficios para los trabajadores son prioritarios como base laboral, por lo que se debe estar apegado a la ley y sus condiciones en las que el trabajador no puede ser degradado.

En este orden de ideas, se puede afirmar que las condiciones de trabajo se ajustan en la medida de la realidad, a partir del conocimiento, experiencia del trabajador y estableciendo actividades de entrega puntual y proporcionales a la importancia de los servicios prestados y otorgados de forma equitativa a los trabajadores; en efecto la Unión General de Trabajadores (2011) se apega a lo expuesto ajustándose a la innovadora mecanización del trabajo, los cambios de ritmo, de producción, los horarios, las tecnologías y aptitudes personales.

\section{Condiciones Físicas}

Es preciso hacer mención, a este aspecto, considerando que las medidas tomadas frente a la pandemia por COVID-19 han hecho que la mayoría de las personas tengan que laborar de forma digital y remota; de este modo, para la Organización Internacional del Trabajo (OIT, 2014) las comodidades en los espacios de trabajo son prioridad, entre otros aspectos, al tener que lograr una uniformidad en estas. La importancia de este aspecto la confirman Barbany, Bieniarz, Carranza, Fuster y otros (1986; Citado por Escalante y Pilar 2012) quienes consideran que la motricidad del trabajador es susceptible de mejora por medio de trabajo físico; por su parte Orlando (2019) opina que el grado de desarrollo que tiene los trabajadores a nivel físico y motriz son relevantes. Sin embargo, las empresas a nivel general no consideran estos aspectos, ya que en muchas ocasiones están activamente funcionales de forma remota, teniendo en cuenta que este tipo de actividades trae inconvenientes en algunos casos como el sedentarismo, contracturas musculares y mala circulación, aspectos físicos que se oponen a lo propuesto por los autores en sus conceptos.

\section{Condiciones temporales}

El estado de alarma ocasionado por la emergencia sanitaria ha ocasionado un impacto, donde las restricciones afectan en la postura del trabajador, ya que los procesos de suspensiones, cierres de empresas, horarios rotativos regulados por la cuarentena, han causado un desajuste en el proceso contractual del personal. Para Salazar et. al. (2017) "la actividad humana discurre en el tiempo, ya que este existe independientemente de nuestra voluntad, es continuo y discurre irreversiblemente, de tal manera que el tiempo perdido jamás vuelve” (pág. 54). 
En tal sentido, todas las medidas dictadas crean un evento de fuerza mayor, teniendo un impacto en las relaciones laborales, donde la temporalidad es una alternativa autorizada por la crisis. En este caso, Rodríguez y López (2015) revelan la adaptación al teletrabajo y la necesidad de, a través de la tecnología, mantener los productos y servicios en el mercado entre otras medidas que han sometido las empresas derivadas de los cambios en el entorno empresarial, como la aceleración de los mercados, las innovaciones tecnológicas, lo que indudablemente, afecta en gran medida la calidad del empleo una empresa en una situación excepcional.

Por lo expuesto, es ineludible considerar la realidad y los derechos humanos, dando garantía a su estabilidad, en búsqueda de la obligatoriedad del deber de las organizaciones para con sus trabajadores; al respecto, la Organización Internacional del Trabajo (OIT, 2014) se apega a que los trabajadores son contratados sólo por un período de tiempo específico, incluye los contratos de duración determinada, así como el trabajo ocasional o estacional, incluido el trabajo por días, que le permita a los trabajadores obtener beneficios sociales para el equilibrio familiar.

\section{Condiciones Psicológicas}

La pandemia del COVID-19 ha cambiado la forma de trabajar, indudablemente estén en casa o en la oficina las personas están presentando miedos, ansiedad, emociones fuertes que ocasionan enfermedades que les pueden atropellar su estabilidad emocional. Según Jiménez (2020) los puestos de trabajo cuando no se apegan a la necesidad del empleado producen tedio, monotonía, fatiga y naturalmente, merma de la productividad, y si se le suma la crisis sanitaria el riesgo para la salud emocional de los afectados es mayor.

Para la Organización Mundial de la Salud OMS (2016) el aspecto psicológico es un componente integral y esencial de la salud, y sobre esta señala que es un estado de bienestar físico, mental y social, y no simplemente la ausencia de afecciones o enfermedades. En efecto, es crítico en esta pandemia que se pueda reconocer los signos de estrés, se considera necesario tomar medidas resilientes para el control de la situación. Según Emiyo, et. al. (2016) existe variedad de condiciones, pero las psicológicas nos orientan para obtener un equilibrio mental, donde se debe involucrar la voluntad, motivación e intereses, confianza, solución de problemas y autocontrol emocional.

\section{Condiciones de seguridad}

Las consecuencias a nivel global por la pandemia del COVID-19, repercuten en las condiciones de seguridad de todos, es así como, a nivel empresarial se toman medidas que priorizan la salud de los trabajadores. Para ello les proporcionan equipos de protección, se aplican mediadas de distanciamiento, horarios rotativos de presencialidad y manejo de procesos virtuales en su mayoría; bajo esta situación, Jiménez (2020) considera que las condiciones de seguridad son el conjunto de medidas técnicas, educativas, médicas y psicológicas empleadas, como formas adecuadas para mantener la estabilidad empresa-trabajador y así lograr el desarrollo satisfactorio del trabajo.

En tal sentido, Apaza (2012) plantea que evitar la exposición al virus, la protección, seguridad, salud y bienestar de las personas involucradas en el trabajo generan una gestión adecuada a nivel sanitario, apoyado por Cisnero (2015) el cual se orienta a una propuesta para crear las condiciones para que el trabajador pueda desarrollar su labor de modo eficiente y sin riesgos, en pocas palabras disfrutando condiciones de seguridad favorables.

\section{Accidentes Laborales}

Según Botta (2018) los accidentes en el trabajo o área laboral son los que se presentan de forma aleatoria, son impredecibles, no es posible saber cuándo van a suceder; mientras que, para Yepez (2015) son lesiones 
funcionales producidas en ejercicio o con motivo del trabajo. De esta manera, en el contexto de la pandemia la seguridad y salud ocupacional adquiere una postura relevante, siendo un aspecto fundamental en el trabajo decente, ya que, en su esencia, vela por la prevención de esos accidentes laborales que tanto perjudican al trabajador, a la organización y hasta la familia en ciertas ocasiones.

El accidente de trabajo representa las evidencias de las malas condiciones de trabajo o dado su nivel de frecuencia. Medioambientales (AEAFMA-IRF España, 2019) define la lesión corporal que el trabajador sufra con ocasión o a consecuencia del trabajo o por cuenta ajena debe aminorarse, es por ello, que las medidas consideradas en la actual pandemia mitigan los riesgos de salud, incluyendo los derivados de las nuevas formas de trabajo.

\section{Medidas Preventivas}

Los accidentes del trabajo y las enfermedades a nivel profesional repercuten en los trabajadores y familiares, en todos los niveles, afectando a las empresas a nivel productivo, ocasionando cierres, paralizaciones temporales, es por ello, que Saldaña Lusarreta (2012, Citado por Arce 2017) se basa en identificar las condiciones de trabajo desfavorables en la búsqueda de mejoramiento progresivo de las acciones internas y la proyección a nivel de servicio, así como también en intentar eliminar dichas condiciones desfavorables. Para ello evalúa los riesgos laborales que no hayan podido ser eliminados, buscando programar correcciones para mejorar las condiciones de seguridad y salud de los trabajadores presentes, mediante medidas que avanzan en el proceso y controlando las correcciones.

Ahora bien, con el propósito de evitar afectaciones, Torres (2015) considera que una decisión evita que aparezca una situación no deseada que podría ocurrir, mientras que la ISO 9000 (2015) plantea que la acción tomada para eliminar la causa, es una situación potencial no deseable. En consecuencia, el control del peligro y riesgo con la adopción de medidas técnicas son alternativas relevantes en la prevención de cualquier situación presente en las empresas incluyendo la del COVID-19, por lo tanto, las medidas preventivas deben ser tomadas a tiempo, por lo que es necesario que en las organizaciones se apliquen estas, para que realmente la prevención sea efectiva y beneficiosa para todos los vinculados.

\section{Enfermedades Ocupacionales}

Desde el inicio de la pandemia existe la preocupación de incluir la COVID-19, como una enfermedad ocupacional, sin embargo, puede considerarse que es contraída por el trabajo o condiciones generadas en el medio ambiente de trabajo. Para tal efecto, se han establecido regímenes de seguridad sanitaria con el fin de manejar un proceso transitorio a nivel de seguridad social, en este sentido, el artículo $4^{\circ}$ de la Ley 1562 de 2012 define como enfermedad laboral aquella que es contraída como resultado de la exposición a factores de riesgo inherentes a la actividad laboral o del medio en el que el trabajador se ha visto obligado a trabajar. Por otra parte, Cáceres (2008) citado por Pérez (2014) define las enfermedades ocupacionales como estado patológico contraído con exposición al medio en el que el trabajador se encuentra obligado a trabajar.

\section{MÉTODO}

El presente avance de investigación se define como un estudio cuantitativo de tipo descriptivo, su propósito se orienta a recopilar datos y así determinar los aspectos necesarios para analizar las condiciones de trabajo en la pequeña y mediana empresa retos del trabajo decente en una economía en pandemia para la generación de valor en la comunidad laboral que integran las MiPymes del sector servicios en Colombia, para ello se detallarán características sobre los hechos objeto de estudio. Según, Tamayo y Tamayo (2001, p. 46), las investigaciones 
descriptivas comprenden la descripción, registro, análisis e interpretación de la naturaleza actual del problema investigado, además de la composición o proceso del fenómeno.

La muestra seleccionada fue aleatoria dentro de la población logrando hasta la fecha una participación de treinta Mipymes del sector servicios en la ciudad de Bogotá. Igualmente se diseñó un instrumento el cual dispone dieciocho ítems para describir las condiciones de trabajo respondiendo a una escala Likert cuyas opciones son siempre, casi siempre, a veces, nunca y casi nunca, el mismo responde a una confiabilidad de 0,9 según el método de alfa de Cronbach y fue validado por tres expertos en el área, para proceder al análisis estadístico de los resultados por medio de la estadística descriptiva y medidas de tendencia central. Para de esta manera lograr el análisis de la información recolectada y configurar las propuestas pertinentes para la elaboración de estrategias que logren mejorar la situación actual.

\section{RESULTADOS}

A continuación, se presenta el análisis y discusión de los resultados obtenidos. Al aplicar el instrumento a las 30 primeras empresas se pudo observar la tendencia del cuestionario contentivo de 18 (dieciocho) ítems orientados a la medición de la dimensión Condiciones de trabajo con alternativas de respuesta escala likert con cinco alternativas de respuestas: siempre (5), casi siempre (4), algunas veces (3), casi nunca (2) y nunca (1).

En cuanto al indicador condiciones físicas el cual trata de configurar la situación actual de los espacios dispuestos para la labor diaria se diseñaron tres ítems los cuales se presentan a continuación en la tabla 1.

\begin{tabular}{|c|c|c|c|c|c|c|c|c|c|c|c|c|}
\hline \multirow[b]{3}{*}{ ÍTEMS } & \multicolumn{2}{|c|}{$S$} & \multicolumn{2}{|c|}{ CS } & \multicolumn{2}{|c|}{ A } & \multicolumn{2}{|c|}{$\mathrm{CN}$} & \multicolumn{2}{|c|}{$N$} & & \\
\hline & & npre & & & & eces & Cas & Vunca & & & & \\
\hline & FA & $\mathrm{FR} \%$ & FA & $\mathrm{FR} \%$ & FA & FR\% & FA & FR\% & FA & FR\% & FA & FR\% \\
\hline $\begin{array}{l}\text { 1. Las condiciones físicas de trabajo son las idóneas } \\
\text { para el desarrollo de las actividades de los } \\
\text { colaboradores dentro de la empresa }\end{array}$ & 14 & 47 & 10 & 33 & 1 & 3 & 5 & 17 & 0 & 0 & 30 & $100 \%$ \\
\hline $\begin{array}{l}\text { 2. En el lugar de trabajo manejan estándares de } \\
\text { seguridad }\end{array}$ & 13 & 43 & 17 & 57 & 0 & 0 & 0 & 0 & 0 & 0 & 30 & $100 \%$ \\
\hline $\begin{array}{l}\text { 3. Se mantiene una buena ventilación e iluminación } \\
\text { en los puestos de trabajo }\end{array}$ & 10 & 33 & 20 & 67 & 0 & 0 & 0 & 0 & 0 & 0 & 30 & $100 \%$ \\
\hline PROMEDIO & $\begin{array}{r}12, \\
3\end{array}$ & 41 & 15,7 & 52,3 & 0,3 & 1 & 1,7 & 5,7 & 12,3 & 41 & & $100 \%$ \\
\hline
\end{tabular}

Tabla 1. Indicador: Condiciones Físicas. Fuente: Torres, Molero, Venegas 2020.

Al interpretar los resultados para el indicador condiciones físicas , para el ítem 1, en cuanto a si las condiciones físicas de trabajo son las idóneas para el desarrollo de las actividades de los colaboradores dentro de la empresa, el $47 \%$ respondió que sí solo el $17 \%$ respondió casi nunca por lo que se puede inferir que las empresas consultadas brindan condiciones físicas suficientes para el desarrollo de las actividades diarias de los colaboradores, en relación con el ítem 2 referente a los estándares de seguridad el $43 \%$ respondió que no hubo respuestas negativas, el $57 \%$ consideró que casi siempre se manejan estándares de calidad en sus empresas.

Continuando con la descripción en relación con la ventilación y la iluminación un 67\% respondió casi siempre para estas condiciones por lo que es necesario encaminar estrategias para atender estos elementos que son de gran importancia para el desarrollo de las funciones de los colaboradores. Referente a estos resultados la OIT (2014) es enfática en relación con que las comodidades en los espacios de trabajo son prioridad, entre otros aspectos, son de vital importancia para que los individuos logren desarrollar sus actividades de manera productiva, sobre todo en la actualidad donde el trabajo en casa es una realidad para miles de personas en el mudo. 


\begin{tabular}{|c|c|c|c|c|c|c|c|c|c|c|c|c|}
\hline \multirow[b]{3}{*}{ ÍTEMS } & \multicolumn{2}{|c|}{$S$} & \multicolumn{2}{|c|}{ CS } & \multicolumn{2}{|c|}{$A$} & \multicolumn{2}{|c|}{$\mathrm{CN}$} & \multicolumn{2}{|c|}{$\mathrm{N}$} & & \\
\hline & & mpre & Casi & iempre & & eces & & Nunca & & Inca & & TAL \\
\hline & $\mathrm{FA}$ & $\mathrm{FR} \%$ & FA & FR\% & FA & $\mathrm{FR} \%$ & $\mathrm{FA}$ & $\mathrm{FR} \%$ & FA & $\mathrm{FR} \%$ & FA & FR\% \\
\hline $\begin{array}{l}\text { 4. Las actividades asignadas a los } \\
\text { colaboradores requieren cumplir una jornada } \\
\text { normal de trabajo }\end{array}$ & 16 & 53 & 7 & 23 & 7 & 23 & 0 & 0 & 0 & 0 & 30 & $100 \%$ \\
\hline $\begin{array}{l}\text { 5. El cumplimiento de las labores de los } \\
\text { colaboradores requiere Trabajar horas extras }\end{array}$ & 14 & 47 & 7 & 23 & 4 & 13 & 0 & 0 & 5 & 17 & 30 & $100 \%$ \\
\hline $\begin{array}{l}\text { 6. Existe un balance entre las exigencias } \\
\text { laborales con respecto a las normas laborales } \\
\text { vigentes }\end{array}$ & 3 & 10 & 6 & 20 & 9 & 30 & 7 & 23 & 5 & 17 & 30 & $100 \%$ \\
\hline PROMEDIO & 11 & 37 & 6,7 & 22 & 6,7 & 22 & 2,3 & 7,7 & 3,3 & 11,3 & & $100 \%$ \\
\hline
\end{tabular}

Tabla 2. Indicador: Condiciones Temporales. Fuente: Torres, Molero, Venegas 2020.

En cuanto a los resultados del indicador "Condiciones Temporales", se puede observarse que el ítem 4 referido a si las jornadas de trabajo se desarrollan en el tiempo normal establecido un $53 \%$ respondió que si se desarrollan en esos tiempos. Para el ítem 5, referente a las horas extras un $47 \%$ respondió que siempre y un $17 \%$ que nunca lo que se contradice con el ítem 8 y pone en evidencia las extensas jornadas de trabajo, el ítem 6 , referente a la existencia del balance entre las exigencias laborales y las normas laborales un $30 \%$ contestó que a veces y solo un $10 \%$ siempre lo que expresa la debilidad del indicador.

\begin{tabular}{|c|c|c|c|c|c|c|c|c|c|c|c|c|}
\hline \multirow[b]{3}{*}{ ÍTEMS } & \multicolumn{2}{|c|}{$S$} & \multicolumn{2}{|c|}{ CS } & \multicolumn{2}{|c|}{$A$} & \multicolumn{2}{|c|}{$N$} & \multicolumn{2}{|c|}{$C N$} & & \\
\hline & \multicolumn{2}{|c|}{ Siempre } & \multicolumn{2}{|c|}{$\begin{array}{c}\text { Casi } \\
\text { siempre }\end{array}$} & \multicolumn{2}{|c|}{ A veces } & \multicolumn{2}{|c|}{ Nunca } & \multicolumn{2}{|c|}{ Casi Nunca } & \multicolumn{2}{|c|}{ TOTAL } \\
\hline & FA & $\mathrm{FR} \%$ & FA & $\mathrm{FR} \%$ & FA & $\mathrm{FR} \%$ & FA & FR\% & FA & $\mathrm{FR} \%$ & FA & $\mathrm{FR} \%$ \\
\hline $\begin{array}{l}\text { 7. Las actividades asignadas a los colaboradores } \\
\text { propician el aburrimiento o desmotivación en su trabajo }\end{array}$ & 16 & 53 & 9 & 30 & 5 & 17 & 0 & 0 & 0 & 0 & 30 & $100 \%$ \\
\hline $\begin{array}{l}\text { 8. Las labores asignadas propician la monotonía en el } \\
\text { lugar de trabajo }\end{array}$ & 4 & 13 & 3 & 10 & 5 & 17 & 11 & 37 & 7 & 23 & 30 & $100 \%$ \\
\hline $\begin{array}{l}\text { 9. El cumplimiento de las labores propicia la fatiga en el } \\
\text { lugar de trabajo }\end{array}$ & 4 & 13 & 5 & 17 & 4 & 13 & 10 & 34 & 7 & 23 & 30 & $100 \%$ \\
\hline PROMEDIO & 8 & 26 & 5,7 & 19 & 5 & 16 & 7 & 24 & 4,7 & 15 & 30 & $100 \%$ \\
\hline
\end{tabular}

Tabla 3. Indicador: Condiciones Psicológicas. Fuente: Torres, Molero, Venegas 2020.

En lo que respecta al indicador condiciones psicológicas se señalan los resultados en la tabla 3. Estos resultados se obtuvieron sobre la base de los promedios obtenidos para los ítems 7 al 9. Para el ítem 7 referente al aburrimiento y desmotivación el $53 \%$ respondió siempre y el $13 \%$ respondió que casi nunca evidenciando la desmotivación generada por las actividades realizadas, así mismo para el ítem 8 referente a la monotonía un 37\% contestó que casi nunca y solo el 13\% respondió que siempre. Para concluir el ítem 9 referente a la fatiga el 34\% contesto casi nunca y el 13\% siempre. En relación con este indicador según Jiménez (2020) los puestos de trabajo, cuando no se apegan a la necesidad del empleado producen tedio, monotonía, fatiga y naturalmente, merma de la productividad, y si se le suma la crisis sanitaria mayor es el riesgo presente en la salud emocional de los afectados.

\begin{tabular}{|c|c|c|c|c|c|c|c|c|c|c|c|c|}
\hline \multirow[b]{3}{*}{ ÍTEMS } & \multicolumn{2}{|c|}{$S$} & \multicolumn{2}{|c|}{ CS } & \multicolumn{2}{|c|}{$A$} & \multicolumn{2}{|c|}{$\mathrm{N}$} & \multicolumn{2}{|c|}{$\mathrm{CN}$} & & \\
\hline & & npre & & $\begin{array}{l}\text { asi } \\
\text { npre }\end{array}$ & & eces & & Inca & & Vunca & & TAL \\
\hline & FA & FR\% & FA & $\mathrm{FR} \%$ & FA & $\mathrm{FR} \%$ & FA & FR\% & $\mathrm{FA}$ & FR\% & FA & FR\% \\
\hline $\begin{array}{l}\text { 10. Los empleados han sufrido accidentes o } \\
\text { incidentes dentro del lugar de trabajo }\end{array}$ & 3 & 10 & 4 & 13 & 6 & 20 & 10 & 33 & 7 & 23 & 30 & $100 \%$ \\
\hline $\begin{array}{l}\text { 11. Los actos inseguros son causa de los accidentes } \\
\text { ocurridos en el lugar de trabajo }\end{array}$ & 2 & 7 & 4 & 13 & 4 & 13 & 10 & 33 & 10 & 33 & 30 & $100 \%$ \\
\hline $\begin{array}{l}\text { 12. Las condiciones inseguras son la causa de los } \\
\text { accidentes ocurridos en el lugar de trabajo }\end{array}$ & 1 & 3 & 5 & 17 & 4 & 13 & 8 & 27 & 12 & 40 & 30 & $100 \%$ \\
\hline
\end{tabular}




\begin{tabular}{|c|c|c|c|c|c|c|c|c|c|c|c|c|}
\hline $\begin{array}{l}\text { 13. La empresa comunica las medidas de } \\
\text { prevención de accidentes en el lugar de trabajo }\end{array}$ & 2 & 7 & 4 & 13 & 6 & 20 & 7 & 23 & 11 & 37 & 30 & $100 \%$ \\
\hline $\begin{array}{l}\text { 14. La empresa divulga la localización de los riesgos } \\
\text { en el lugar de trabajo }\end{array}$ & 16 & 53 & 3 & 10 & 7 & 23 & 4 & 13 & 0 & 0 & 30 & $100 \%$ \\
\hline $\begin{array}{l}\text { 15. La empresa informa la utilización de los equipos } \\
\text { de seguridad en el lugar de trabajo }\end{array}$ & 18 & 60 & 6 & 20 & 2 & 7 & 4 & 13 & 0 & 0 & 30 & $100 \%$ \\
\hline $\begin{array}{l}\text { 16. Los colaboradores han sufrido alguna } \\
\text { enfermedad producto del desarrollo de sus } \\
\text { actividades en la empresa }\end{array}$ & 21 & 70 & 5 & 17 & 4 & 13 & 0 & 0 & 0 & 0 & 30 & $100 \%$ \\
\hline $\begin{array}{l}\text { 17. La empresa practica periódicamente exámenes } \\
\text { médicos para conocer su estado de salud }\end{array}$ & 0 & 0 & 0 & 0 & 6 & 20 & 7 & 23 & 17 & 57 & 30 & $100 \%$ \\
\hline $\begin{array}{l}\text { 18. Verifican el seguimiento de normas en salud } \\
\text { ocupacional }\end{array}$ & 17 & 57 & 7 & 23 & 5 & 17 & 1 & 3 & 0 & 0 & 30 & $100 \%$ \\
\hline PROMEDIO & 8,9 & 30 & 4,2 & 14 & 4,9 & 16 & 5,7 & 19 & 6,3 & 21 & & \\
\hline
\end{tabular}

En cuanto a los resultados para el subdimensión condiciones de seguridad, pude observarse que los ítems 10,11 y 12 referentes al indicador accidentes de trabajo la opción nunca fue la que mostró porcentajes más altos, sin embargo, entre las opciones casi siempre y a veces se alcanza un 30\%. En relación con los ítems 13, 14 y 15 referentes al indicador medidas preventivas, los porcentajes más representativos fueron los referentes a la comunicación de las condiciones inseguras y las medidas de prevención siendo de 40\% y 37\% para las opciones nunca. Por último, los ítems 16,17 y 18 enfocados en las enfermedades ocupacionales, el 57\% refirió que nunca se realizan exámenes periódicos, sin embargo, el 70\% respondió no sufrir de enfermedades ocupacionales.

\section{CONCLUSIONES}

Este avance de investigación ofrece una visión de las condiciones de trabajo actuales en las empresas consultadas y al confrontar estas con la situación actual para de esta manera exponer la realidad de las mimas.

En lo que corresponde a las condiciones físicas a pesar de ofrecer en su mayoría condiciones aceptables, en la actualidad la situación planteada por la pandemia tiene un efecto diferenciador ya que el trabajo en el hogar modifica las condiciones de trabajo habituales y las existentes para las personas que están trabajando de manera presencial aún presentan un porcentaje que debe mejorarse para lograr un ambiente de trabajo más productivo.

Así miso en relación con las condiciones temporales es de gran importancia analizar el exceso de horas extras que se están manejando a pesar de la virtualidad, es uno de los elementos más visibles en el trabajo a distancia ya que no se maneja un horario de trabajo por lo que no se logra diferenciar las horas laborales y las horas de descanso.

Continuando con las reflexiones finales se destacan las condiciones psicológicas las cuales son unas de las más afectadas debido al escenario actual, destacando la relación del estrés y la monotonía del trabajo el cual representa una de las situaciones recurrentes en las empresas consultadas.

Finalmente, en lo que corresponde a las condiciones de seguridad es de gran importancia reforzar cada elemento que configura esta dimensión para responder a las exigencias actual y para atender la salud de los trabajadores en cada uno de los escenarios aborales sobre todo en un sector tan vulnerable. Así mismo avanzar en investigación es que estudien las distintas perspectivas de sus dimensiones dentro del ámbito laboral. 


\section{REFERENCIAS BIBLIOGRÁFICAS}

Apaza, R. (2012). Seguridad y salud ocupacional: Definición. Recuperado de: https://www.rubenapaza.com/2012/12/seguridad-y-salud-ocupacional-definicion.html

Arce, S. (2017) La prevención de riesgos laborales y accidentalidad laboral en la prensa española: representación y cobertura a partir de la Ley 31/1995 de prevención de riesgos laborales (1994-2014). Universidad de Burgos. Recuperado de: https://dialnet.unirioja.es/servlet/tesis?codigo=105848

Asociación Española de Agentes Forestadles y Medioambientales (AEAFMA-IRF España)(2019) Agentes Forestales. Amparo a la Ley Orgánica del 2002. Recuperado de: http://agentesforestales.org/aeafma/99-aeafma.html

Ballesteros, C. (2016). El trabajo decente:¿la aspiración del siglo XXI?. Facultad de ciencias sociales, jurídicas y de la comunicación. Universidad de Valladolid. Recuperado de: https://core.ac.uk/download/pdf/211101664.pdf

Botta, N. (2018). Los accidentes de trabajo. Editorial Proteger. Enero 2018. Material no acto para la venta. Seguridad e Higiene en el trabajo. Recuperado de:

https://www.redproteger.com.ar/editorialredproteger/serieaccidentologia/67_Los_Accidentes_Trabajo _2a_edicion_enero2018.pdf

CEPAL. (2020). 4 Informe: Sectores y empresas frente al COVID-19: emergencia y reactivación. CEPAL. Recuperado de: https://repositorio.cepal.org/bitstream/handle/11362/45734/4/S2000438_es.pdf

Cisneros, C. (2015).Los accidentes laborales, su impacto económico y social. Ciencia Holguin. Vol XXI, num 3. Julio-septiembre,2015.pp. 1-11. Centro de información y gestión tecnológica de Santiago de Cuba Hoguin Cuba. Recuperado de: https://www.redalyc.org/pdf/1815/181541051002.pdf

Ley 1562 (2012). Por la cual se modifica el sistema de riesgos laborales y Se dictan otras disposiciones en materia de salud Ocupacional. Congreso de Colombia. Recuperado de: https:/www.minsalud.gov.co/sites/rid/Lists/BibliotecaDigital/RIDE/DE/DIJ/Ley-1562-de-2012.pdf

Emiyo, J., Idrogo, A., Padrón, A y Sequea, A (2016). Condiciones Psicológicas y ambientales del estudio. Recuperado de: https://es.scribd.com/presentation/280965341/Condiciones-Psicologicas-Del-Trabajo

Escalante L y Pila, H. (2012); La condición física. Evolución histórica de este Concepto. Lectura: Educación Física y Deporte. Revista Digital. Buenos Aires. Recuperado de: https://revistas.udca.edu.co/index.php/rdafd

Gómez P., Calderón (2012). Salud mental en el trabajo: entre el sufrimiento en el trabajo y la organización saludable. Katharsis, N 23. Recuperado de:

http://revistas.iue.edu.co/revistasiue/index.php/katharsis/article/view/871

Organización Mundial de la Salud OMS (2016). Condiciones de Trabajo. Recuperado de: http://www.scielo.org.co/pdf/rups/v14nspe5/v14nspe5a07.pdf

Jiménez (2020). Condiciones Psicológicas del trabajo. Revista Laboral. Recuperado de: https://dialnet.unirioja.es/descarga/libro/682131.pdf

Larani, M.E. (2015); Trabajo decente como eje de equidad una nueva agenda en las políticas sociales. 
Una nueva agenda de la política. Recuperado de: https://core.ac.uk/download/pdf/11677028.pdf

Monasterio Villamizar y Asociados (2015). Condiciones de trabajo. La ley Federal del Trabajo. Jornada laboral. Recuperado de:

https://definicionlegal.blogspot.com/2015/05/condiciones-de- trabajo.html

Organización Internacional del Trabajo (OIT) (2014); Educación obrera para el trabajo decente. Condiciones de trabajo. Oficina de País para la Argentina. Oficina de actividades para los trabajadores (ACTRAV).

Orlando (2019). Condición física y salud. Mundo de entrenamiento el deporte bajo evidencia Científica. Todo sobre la educación física. El deporte bajo evidencia científica. Mundo. Recuperado de: https://mundoentrenamiento.com/condicion-fisica-y-salud/

Pérez, J. (2014). Riesgo laboral y su incidencia en las enfermedades ocupacionales de los trabajadores de la brigada motorizada de la policía del Estado Carabobo. Recuperado de:

http://mriuc.bc.uc.edu.ve/bitstream/handle/123456789/5964/iperez.pdf?sequence=1

Rodríguez, E y López, L. (2015). El empleo temporal en la industria colombiana 200-2013. Universidad de Medellín. Recuperado de: https://dialnet.unirioja.es/descarga/articulo/5607266.pdf

Salazar, V., Carreño, V., García, G., Agüero , P y Apestegui, M. (2017). Manual de métodos de estudio. Facultad de Ciencias Administrativas y Recursos Humanos. Facultad de Ciencias Contables Económicas y Financieras. Ciudad Universitaria USMP. Lima. Recuperado de: https://www.usmp.edu.pe/estudiosgenerales/pdf/2017-I/MANUALES/METODOS\%20DE\%20ESTUDIO.pdf

Torres, I (2015). Acción preventiva qué es y por qué ya no aparece en ISO 9001? Recuperado de https://iveconsultores.com/accion-preventiva/

Unión general de trabajadores (2011). Prevención de riesgos laborales, condiciones de trabajo UGT . Recuperado de: http://portal.ugt.org/campanas/condicionesdetrabajo.pdf

Yépez, A. (2015). Accidentes y riesgos de trabajo. Recuperado de: http://www.iparkman.com/es/articulos/derecho-laboral/2361-autor-araceli-yepez-roa-accidentes-y-riesgos-de-trabajo 